\title{
Synthesis of Magnetite Nanoparticles by Top-Down Approach from a High Purity Ore
}

\author{
Gayan Priyadarshana, ${ }^{1}$ Nilwala Kottegoda, ${ }^{1,2}$ Atula Senaratne, ${ }^{3}$ \\ Ajith de Alwis, ${ }^{4}$ and Veranja Karunaratne ${ }^{1,5}$ \\ ${ }^{1}$ Sri Lanka Institute of Nanotechnology (SLINTEC), Nanotechnology \& Science Park, Mahenawatta, Pitipana, \\ 10200 Homagama, Sri Lanka \\ ${ }^{2}$ Department of Chemistry, University of Sri Jayewardenepura, 10250 Nugegoda, Sri Lanka \\ ${ }^{3}$ Department of Geology, University of Peradeniya, 20400 Peradeniya, Sri Lanka \\ ${ }^{4}$ Department of Chemical and Process Engineering, University of Moratuwa, 10400 Moratuwa, Sri Lanka \\ ${ }^{5}$ Department of Chemistry, University of Peradeniya, 20400 Peradeniya, Sri Lanka
}

Correspondence should be addressed to Nilwala Kottegoda; nilwalak@slintec.lk

Received 11 June 2015; Revised 26 August 2015; Accepted 9 September 2015

Academic Editor: Rajender S. Varma

Copyright ( 2015 Gayan Priyadarshana et al. This is an open access article distributed under the Creative Commons Attribution License, which permits unrestricted use, distribution, and reproduction in any medium, provided the original work is properly cited.

\begin{abstract}
This study attempts to synthesize magnetite nanoparticles from a high purity natural iron oxide ore found in Panvila, Sri Lanka, following a novel top-down approach. Powder X-Ray diffraction, elemental analysis, and chemical analysis data confirmed the ore to be exclusively magnetite with $\mathrm{Fe}^{2+}: \mathrm{Fe}^{3+}$ ratio of $1: 2$. Surface modified magnetite nanoparticles were synthesized by destructuring of this ore using a top-down approach in the presence of oleic acid. These oleic acid coated nanoparticles were further dispersed in ethanol resulting in stable nanomagnetite dispersion. Interestingly, the nanoparticles demonstrated a spherical morphology with a particle size ranging from 20 to $50 \mathrm{~nm}$. Magnetic force microscopic data was used to confirm the topography of the nanoparticles and to study the magnetic domain structure.
\end{abstract}

\section{Background}

Magnetite nanoparticles and their suspensions have shown widespread applications including wastewater treatment [1], heavy metal removal [2-5], magnetic recording devices [6, 7], magnetic data storage devices [8], toners and inks for electrophotography $[9,10]$, magnetic resonance imaging [11], bioseparation, targeted drug delivery [12-15], pigments [10], flocculants [16], and catalytic materials [16, 17].

Numerous methods for synthesis of magnetite nanoparticles have been reported. Among them, the most common method is coprecipitation of ferrous and ferric ions in alkaline medium [6, 13, 18-21]. In addition, magnetite nanoparticles can also be synthesized by thermal decomposition $[19,22]$, sol-gel techniques [23], hydrothermal synthesis [24], electrochemical synthesis [25, 26], microemulsion techniques [27], sonochemical synthesis [28], hydrolysis [29], and microwave assisted synthesis $[26,30]$.
Although chemical precipitation method has been identified as the most economical and practical method for magnetite nanoparticle synthesis, top-down approach involving crushing, milling, or grinding offers a simple environmentally friendly synthetic route despite the long grinding times involved [31]. Due to that interest, in very recent years, high energy ball milling has extensively been used to study the effect on the particle size, crystallite size, and lattice strain in metals, metal oxides, metal alloys, and so forth [32-35]. Physicochemical properties of ball milled boron particles under two grinding techniques such as dry and wet grinding were investigated by Jung et al. [36]. They found that the size distribution, morphology, size reduction rate, and the degree of agglomeration of milled boron particles are affected by milling type as the wet milling process produced boron particles with narrower size distribution, smoother morphology, and less amount of agglomerated particles compared to dry milling process. In another study, Razavi-Tousi and Szpunar 
have investigated the effect of ball size on steady state of aluminum powder and efficiency of impacts during milling and they have concluded that, at a given mill speed and ball to powder ratio, a change in balls' size can improve milling efficiency [37]. In addition, there are evidences to prove that the milling parameters such as speed and time have much impact on the crystallite size during the ball milling [38].

Almásy et al. have compared the properties and the structure of nanoparticles synthesized by coprecipitation and wet grinding methods. They have concluded that the magnetite nanoparticles resulted in by wet grinding process demonstrated higher saturation magnetization than those obtained by rapid coprecipitation method [31]. We report herein the synthesis of magnetite nanoparticles from a naturally occurring magnetite ore deposit located in Panvila, Sri Lanka, using a wet grinding approach involving significantly low grinding time.

Among several naturally occurring magnetite deposits in Sri Lanka, Panvila deposit is the purest of all recent discoveries with $93-98 \%$ magnetite and trace amounts of titanium dioxide as rutile (1.3-3.4\%) and other elements [39]. Geologically, Panvila is situated well within the lithological formation of the highland group. The magnetite bands are sandwiched between crystalline highland group rocks running parallel to the Knuckles Massif. Panvila iron ore is found in high crystalline form with an ore size of approximately one million tons of magnetite.

\section{Materials and Methods}

The natural iron oxide (magnetite) samples were collected from Panvila, with the courtesy of Geological Survey and Mines Bureau (GSMB), Sri Lanka. Table 1 shows the physical properties of the ore deposit. The samples were used as recieved with no chemical modifications, unless stated otherwise. Oleic acid, $\mathrm{H}_{2} \mathrm{SO}_{4}, \mathrm{H}_{3} \mathrm{PO}_{4}, \mathrm{HCl}, \mathrm{KMnO}_{4}$, and ethanol used were of analytical grade. Deionized water was used for all the solution preparations.

Mineralogical phase identification of the natural ore and the synthesised samples was carried out using powder X-Ray diffraction (PXRD) technique using Bruker D8 Focus X-Ray powder diffractometer, using $\mathrm{Cu} K \alpha$ radiation $(\lambda=0.154 \mathrm{~nm})$ over a $2 \theta$ range of $15-85^{\circ}$ with a step size of $0.01^{\circ}$ and a step time of $2 \mathrm{~s}$. Morphological studies were carried out using HITACHI SU6600 scanning electron microscope (SEM). The morphology of the samples was studied using the secondary electron mode and scanning transmission mode. The solid samples were mounted on a carbon tape and coated with a thin layer of Au for SEM imaging. A drop of the magnetite dispersion in ethanol was placed on a carbon coated $\mathrm{Cu}$ mesh and dried for STEM analysis.

The elemental composition was recorded for powdered samples using Philips PW 2400 X-Ray Fluorescence Spectrometer (XRF).

Magnetic force microscopic (MFM) imaging was performed using Park Systems, XE-100 atomic force microscope with a magnetic force microscopic attachment. Magnetite dispersion was mounted onto a mica substrate and dried
TABle 1: Mineralogical data of the magnetite deposit from Panvila, Sri Lanka.

\begin{tabular}{lc}
\hline \multicolumn{2}{c}{ Physical properties } \\
\hline Colour & Black \\
Crystallography & Cubic octahedron \\
Hardness & $5.5-6.5$ \\
Specific gravity & $6.0-6.5$ \\
Streak & Black \\
Lustre & Metallic \\
Cleavages & Parallel/octahedron surfaces \\
Surface & Silky \\
\hline
\end{tabular}

at $100^{\circ} \mathrm{C}$ before microscopic observations. The scans were carried out on $X Y$ plane ( $Z$-decoupled) in noncontact mode.

Particle size distribution and the zeta potential of the dispersions were determined using Malvern Zetasizer; nano ZS (size range, $0.6 \mathrm{~nm}-6 \mu \mathrm{m}$ ). Bruker Vertex 80 coupled with Ram-FT module (RAM II) Fourier transform infrared spectrophotometer (FTIR) in the range of 400 to $4000 \mathrm{~cm}^{-1}$ was used to study the functional groups present in resulted oleic acid coated magnetite nanoparticles. The data was measured in transmittance mode using $\mathrm{KBr}$ pellet method with the ratio of $100: 1 \mathrm{KBr}$ to magnetite sample.

2.1. Determination of $\mathrm{Fe}^{2+}: \mathrm{Fe}^{3+}$ Ratio in the Ore. In order to determine the ratio between ferrous $\left(\mathrm{Fe}^{2+}\right)$ to ferric $\left(\mathrm{Fe}^{3+}\right)$ ions in the magnetite ore, $0.25 \mathrm{~g}$ of powdered raw magnetite sample was dissolved in conc. $\mathrm{HCl}\left(10.00 \mathrm{~cm}^{3}\right)$ under an inert atmosphere $\left(\mathrm{N}_{2}\right)$. The stock solution was made up to $250.00 \mathrm{~cm}^{3}$ using deionized water. Syrupy $\mathrm{H}_{3} \mathrm{PO}_{4}\left(5.00 \mathrm{~cm}^{3}\right)$ and $1 \mathrm{~mol} \mathrm{dm}^{-3} \mathrm{H}_{2} \mathrm{SO}_{4}\left(28.00 \mathrm{~cm}^{3}\right)$ were added to the stock solution $\left(25.00 \mathrm{~cm}^{3}\right)$ and titrated with $0.01 \mathrm{~mol} \mathrm{dm}^{-3} \mathrm{KMnO}_{4}$ to determine the $\mathrm{Fe}^{+2}$ content in the ore according to (1). Zinc granules $(\sim 3 \mathrm{~g})$ were added to $30 \mathrm{~cm}^{3}$ of the above stock solution and stirred for $30 \mathrm{~min}$. Then $\mathrm{Fe}^{3+}$ ions will reduce into $\mathrm{Fe}^{2+}$ ions (see (2)). A volume of $25.00 \mathrm{~cm}^{3}$ of the resulting solution was treated with syrupy $\mathrm{H}_{3} \mathrm{PO}_{4}\left(5.00 \mathrm{~cm}^{3}\right)$ and $\mathrm{H}_{2} \mathrm{SO}_{4}\left(28.00 \mathrm{~cm}^{3}\right)$ and titrated with $0.01 \mathrm{~mol} \mathrm{dm}{ }^{-3} \mathrm{KMnO}_{4}$ for the determination of total $\mathrm{Fe}^{2+}$ ions. By that, the ratio between $\mathrm{Fe}^{2+}$ and $\mathrm{Fe}^{3+}$ was calculated:

$$
\begin{aligned}
& 5 \mathrm{Fe}^{2+}(\mathrm{aq})+\mathrm{MnO}_{4}^{-}(\mathrm{aq})+8 \mathrm{H}^{+}(\mathrm{aq}) \\
& \longrightarrow 5 \mathrm{Fe}^{3+}(\mathrm{aq})+\mathrm{Mn}^{2+}(\mathrm{aq})+4 \mathrm{H}_{2} \mathrm{O}(\mathrm{l}) \\
& 2 \mathrm{Fe}^{3+}(\mathrm{aq})+\mathrm{Zn}(\mathrm{s}) \longrightarrow 2 \mathrm{Fe}^{2+}(\mathrm{aq})+\mathrm{Zn}^{2+}(\mathrm{aq})
\end{aligned}
$$

\subsection{Synthesis of Surface Modified Magnetite Nanoparticles.} A sample of $20 \mathrm{~g}$ of magnetite ore (MAG-Ore) was crushed into a powder using a mortar and a pestle resulting in crushed magnetite powder (Mag-Ore-Crush). The resulted magnetite particles were separated from the ore magnetically using a neodymium block magnet, with a 13,000 Gauss magnetic field strength (MAG-Ore-Sep), and were subjected to grinding in the presence of oleic acid $\left(20 \mathrm{~cm}^{3}\right)$ using 
a FRITSCH Planeten-Mikromühle PULVERISETTE 7 premium line grinder in an inert atmosphere $\left(\mathrm{N}_{2}\right)$. The grinding procedure was continued in a sequential manner as explained below:

(a) Sample of MAG-Ore-Sep was ground using $15 \mathrm{~mm}$ size tungsten carbide grinding balls and the resulted sample is MAG-Ole-Grd-1.

(b) MAG-Ole-Grd-1 is further ground using $5 \mathrm{~mm}$ size tungsten carbide balls resulting in MAG-Ole-Grd-2.

(c) MAG-Ole-Grd-2 is further ground using $3 \mathrm{~mm}$ zirconium oxide balls resulting in MAG-Ole-Grd-3.

(d) MAG-Ole-Grd-3 is further ground using $1 \mathrm{~mm}$ zirconium oxide balls resulting in MAG-Ole-Grd-4.

(e) MAG-Ole-Grd-4 is further ground using $0.5 \mathrm{~mm}$ zirconium oxide balls resulting in MAG-Ole-Grd-5.

Each grinding step was continued for $1 \mathrm{hr}$ duration and the grinding was carried out at $700 \mathrm{rpm}$.

Large particles were separated from the oleic acidnanomagnetite dispersion by centrifugation at $9000 \mathrm{rpm}$ using Sigma 3-18 laboratory centrifuge for $30 \mathrm{~min}$ after each step. The particle size and the zeta potential of the MAG-Ole-Grd-5 dispersion were measured using Zetasizer. Ethanol was added dropwise into $1 \mathrm{~cm}^{3}$ of MAG-Ole-Grd-5 dispersion until a clear and stable solution was obtained prior to analyzing the particle size and zeta potential. SEM imaging, STEM imaging, and MFM imaging of MAG-Ole-Grd-5 were carried out for morphological analysis and magnetic structure studies of the resulting magnetite nanoparticles.

\section{Results and Discussion}

3.1. Characterization of the Magnetite Ore (MAG-Ore-Crush). Figure 1(a) shows the PXRD pattern of the crushed magnetite sample obtained from the ore prior to magnetic separation.

The major phase of the ore was identified as magnetite by referring to the ICDD data base (PDF number 01-089-0691). As shown in Figure 1(b), the product obtained after grinding, according to the suggested method, resulted in a solid material rich in magnetite. There are no detectable crystalline impurities in the final product. However, the intensity of major 311 diffraction peak has significantly reduced as a result of reduction in the crystallite size. Referring to the Scherrer formula, crystallite size of the magnetite powder resulting in after final grinding stage (MAG-Ole-Grd-5) was found to be $11 \mathrm{~nm}$, where that of the initial powder sample (Mag-OreCrush) was $36 \mathrm{~nm}$. Therefore, it is noteworthy to mention that the utilization of kinetic energy of the high density balls has affected the crystallite size reduction. Creation of large numbers of linear defects resulting in high dislocation density regions will lead to the reduction in crystallite size. During further milling, the sample volume exhibiting small grains extends throughout the entire specimen, hence decreasing the effective crystallite size [40]. However, the crystallite size of a crystalline material does not necessarily represent the particle size. In other words, a particle is made up with the combination of crystallites with grain boundaries to form polycrystalline material. It is further confirmed by Figure 3(a). Even though the crystallite size is $36 \mathrm{~nm}$

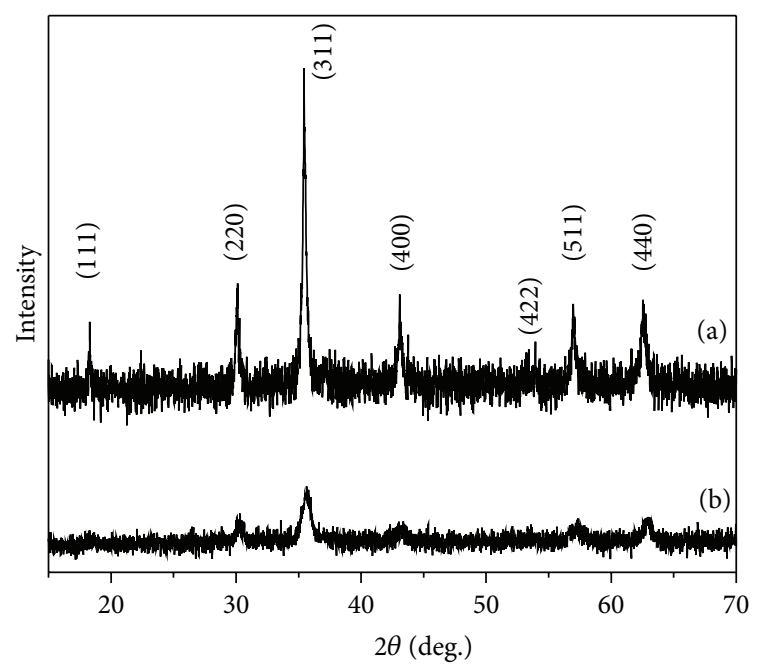

FIGURE 1: PXRD patterns of (a) MAG-Ore-Crush and (b) MAG-OleGrd-5 obtained using Bruker D8 Focus X-Ray powder diffractometer.

according to the Scherrer equation, the particle size of MagOre-Crush in Figure 3(a) is in micron range. Table 2 shows the average percentages of major elements in five replicates of MAG-Ore-Crush. As evidenced by XRF analysis, the ore was rich in iron oxide.

According to the data obtained from the titrimetric method, the molar ratio between $\mathrm{Fe}^{2+}$ and $\mathrm{Fe}^{3+}$ was $1: 2$, which was in agreement with the molar ratio present in the magnetite $\left(\mathrm{Fe}_{3} \mathrm{O}_{4}\right)$.

3.2. Oleic Acid Modified Magnetite Nanoparticles. Oleic acid, a long chain carboxylic acid, was used as a surface modifier during the wet grinding process in order to avoid any particle agglomeration. Unexpectedly, oleic acid coated magnetite nanoparticles with a size distribution of $20-50 \mathrm{~nm}$ were obtained by this approach. In aqueous systems of iron oxides, the surface oxygen atoms bound to Fe atoms undergo protonation with water to form surface hydroxyl groups. Surface hydroxyl groups are amphoteric and may react with either acids or bases. In aqueous dispersions, the surface of magnetite will either be positive or negative, depending on $\mathrm{pH}$ of the solution. In this study, oleic acid molecules react with the surface hydroxyl groups of the magnetite forming a stable coating around nanoparticles, thus preventing their agglomeration.

Particle size analysis obtained from Malvern Zetasizer nano ZS device showed that MAG-Ole-Grd-5 produced a single narrow peak within the range of $20-50 \mathrm{~nm}$ (See Figure 2).

Surprisingly and interestingly, such uniform particle size distribution for stable magnetite nanoparticles synthesized through top-down approach with reasonably reduced grinding time had not been previously reported. The uniform particle size distribution and the size range resulted due to the selection of proper organic medium to stabilize the nanoparticles, the grinding ball size, and ball material. This method 
TABLE 2: Elemental compositions of the MAG-Ore-Crush obtained using Philips PW 2400 X-Ray Fluorescence Spectrometer.

\begin{tabular}{|c|c|}
\hline Element in oxide form & Composition (\%) \\
\hline $\mathrm{SiO}_{2}$ & 0.36 \\
\hline $\mathrm{TiO}_{2}$ & 2.57 \\
\hline $\mathrm{Al}_{2} \mathrm{O}_{3}$ & 2.91 \\
\hline $\mathrm{Fe}_{2} \mathrm{O}_{3}$ & 93.73 \\
\hline $\mathrm{MnO}$ & 0.11 \\
\hline $\mathrm{MgO}$ & 0.20 \\
\hline $\mathrm{CaO}$ & $<0.01$ \\
\hline $\mathrm{Na}_{2} \mathrm{O}$ & $<0.01$ \\
\hline $\mathrm{K}_{2} \mathrm{O}$ & 0.07 \\
\hline $\mathrm{P}_{2} \mathrm{O}_{5}$ & 0.01 \\
\hline
\end{tabular}

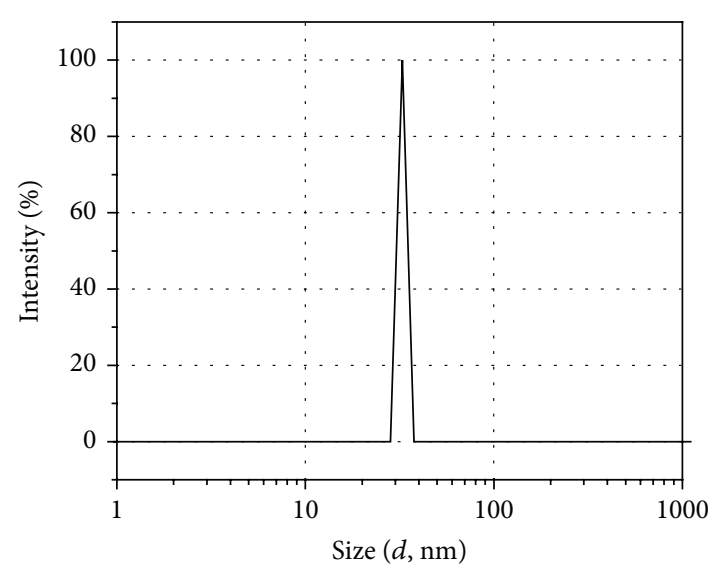

Figure 2: Size distribution of MAG-Ole-Grd-5 dispersed in ethanol obtained using Malvern Zetasizer; nano ZS.

has therefore led to exploration of a commercially viable topdown process for synthesis of magnetite nanoparticles from a natural ore.

SEM images given in Figures 3(a)-3(d) illustrate the particle size and the morphology obtained with grinding balls of different sizes. According to these figures, after the final grinding step with $0.5 \mathrm{~mm}$ zirconia balls (MAG-OleGrd-5), majority of the particles with spherical morphology and particle size less than $50 \mathrm{~nm}$ were produced. These evidences were further confirmed by the STEM image given in Figure 4(a), while Figure 4(b) shows the size distribution of 112 particles in few STEM images and also provides evidence to prove the particle sizes are ranging between $20-50 \mathrm{~nm}$.

Magnetic domain structure and the topography image of the magnetite nanoparticles are shown in Figure 5. As shown in the topography image well defined magnetite particles are observed.

MFM images are formed by scanning the attractive and repulsive magnetostatic field with the interactions between the magnetic tip in vertical direction and the surface of a sample in horizontal position. Magnetic tip represents a magnetic dipole. In the image well-defined walls and domains can be observed where negative shifts (repulsions) appear as dark areas while positive shifts (attractive forces) appear bright in the MFM image. Regular shaped uniformly distributed domains (circular) are observed.

Zeta potential was measured for MAG-Ole-Grd- 5 and the average zeta potential was $+42 \mathrm{mV}$. Generally, for nanoparticles, a high zeta potential confers stability; that is, the solution or dispersion resists forming agglomerated particles. Accordingly, the above stated dispersions are in the highly stable range. It was also observed that these dispersions were stable over six months.

Figure 6(a) shows the FTIR spectrum of the magnetite nanoparticles coated with oleic acid. Here, the characteristic absorption of $\mathrm{Fe}-\mathrm{O}$ bond appeared at $578 \mathrm{~cm}^{-1}$, which is in close agreement with those reported previously $[6,41,42]$. Figure 6(b) shows the FTIR spectrum of pure oleic acid. Two sharp bands appearing at 2923 and $2852 \mathrm{~cm}^{-1}$ were attributed to the asymmetric and symmetric stretching frequencies of $\mathrm{CH}_{2}$ groups of oleic acid, respectively. The intense peak at $1710 \mathrm{~cm}^{-1}$ was due to the presence of $\mathrm{C}=\mathrm{O}$ stretching and the band at $1285 \mathrm{~cm}^{-1}$ was due to $\mathrm{C}-\mathrm{O}$ stretching. In surface modified magnetite nanoparticles (Figure 6(a)), the asymmetric $\mathrm{CH}_{2}$ stretching and the symmetric $\mathrm{CH}_{2}$ stretching had shifted to 2920 and $2850 \mathrm{~cm}^{-1}$, respectively. This observation confirmed that the adsorbed oleic acid molecules are in a closed pack crystalline state as a monolayer arrangement around the magnetite nanoparticles [22, 43]. Moreover, the $\mathrm{C}=\mathrm{O}$ stretching band of the carboxyl group, which occurred at $1710 \mathrm{~cm}^{-1}$ in Figure 6(b), was absent in Figure 6(a). Instead, there were two bands appearing at 1540 and $1410 \mathrm{~cm}^{-1}$ which were characteristic of the asymmetric $v_{\mathrm{as}}\left(\mathrm{COO}^{-}\right)$and the symmetric $\nu_{s}\left(\mathrm{COO}^{-}\right)$stretching [43] revealing that the oleic acid was chemisorbed onto the magnetite nanoparticles as a carboxylate.

It has been reported that, during grinding process, inelastic collisions between the particles and the balls destructure the particles leading to the formation of fresh interfaces between the reaction phases. Such processes involve dynamic deformation, fracturing, and cold welding. The frictional heat thus dissipated over collision areas is transferred from balls to particles that empowered the exposed Fe ions to react with oleic acid molecules. As a result, oleic acid is absorbed onto these freshly appeared surfaces through different modes of bonding such as H-bonding and van der Waals bonding. The successful synthesis of uniform sized magnetite particles by mechanical grinding process could be related particularly to the appropriate selection of the grinding medium, ball size, ball material, and milling time. This observation could be further explained by referring to Almásy et al. [31]. They have concluded that the deformation size in a powder particle that is trapped between balls during high impact milling process could be expressed as a function of the distance from the particle center to the contact area, the relative impact speed, the ball density, and the hardness of the particle [31]. When the grinding was performed using a given ball size at a particular grinding step, it was observed that the particle size reaches an equilibrium where the further grinding with grinding balls of the same size will not affect the size of 


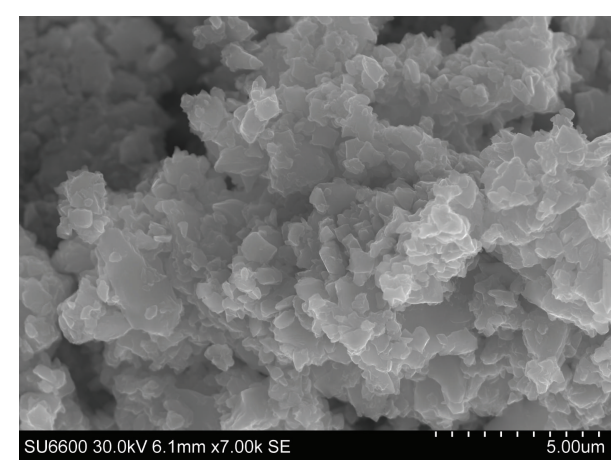

(a)

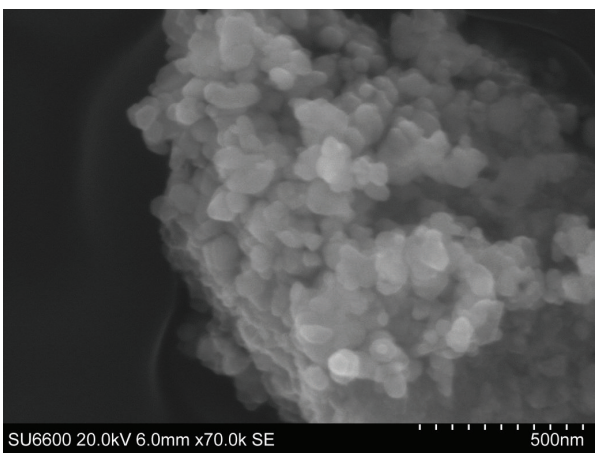

(c)

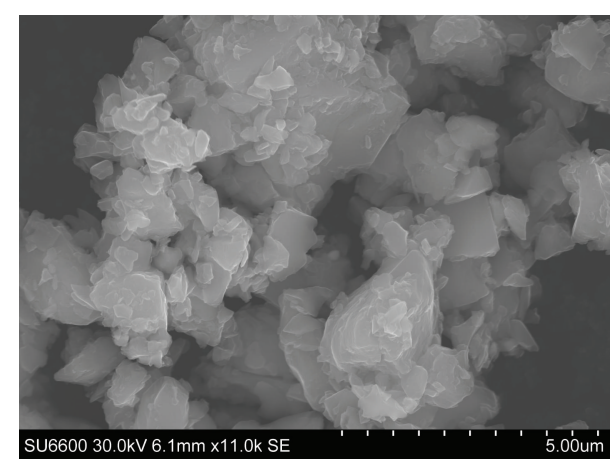

(b)

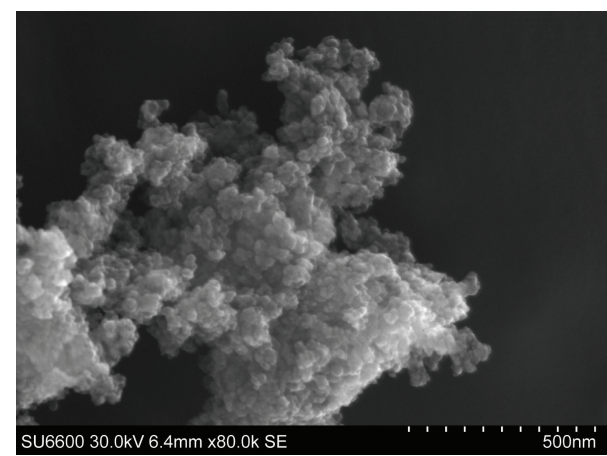

(d)

FIGURE 3: SEM images of magnetite particles after grinding with different sized grinding balls (a) MAG-Ole-Grd-1, (b) MAG-Ole-Grd-2, (c) MAG-Ole-Grd-4, and (d) MAG-Ole-Grd-5 obtained using HITACHI SU6600 scanning electron microscope.

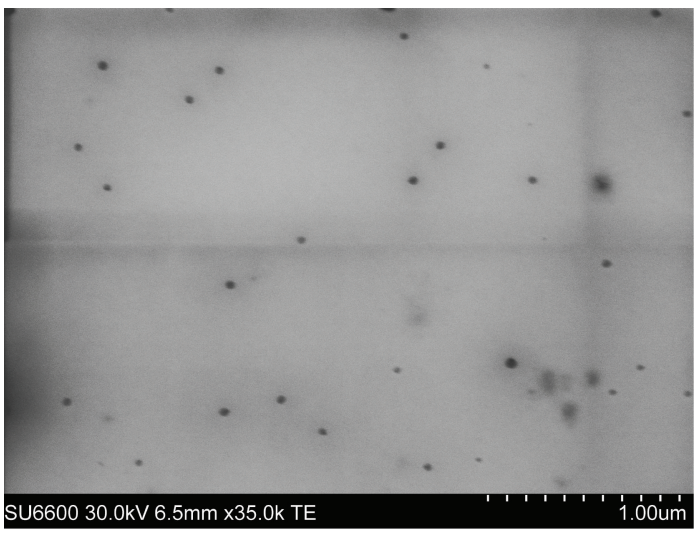

(a)

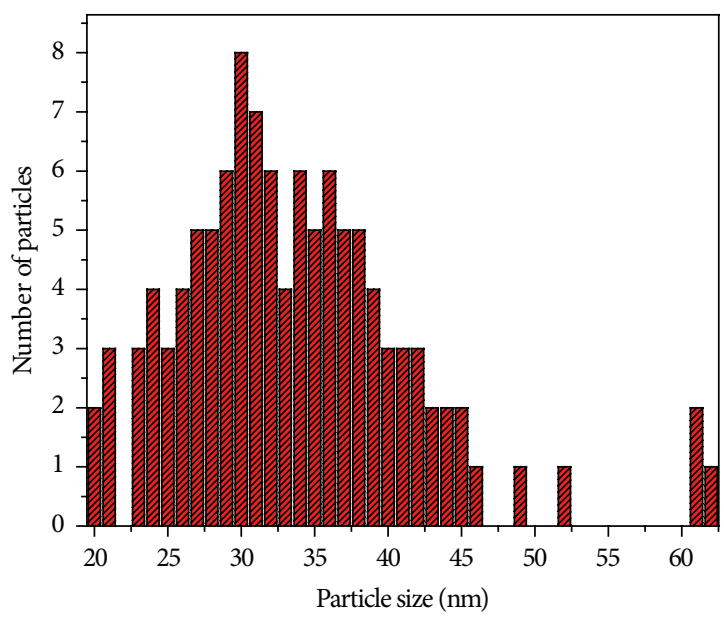

(b)

FIGURE 4: (a) STEM image of MAG-Ole-Grd-5 after being dispersed in ethanol obtained using HITACHI SU6600 scanning electron microscope. (b) Distribution of magnetite nanoparticles of MAG-Ole-Grd-5 tabulated using the particle size of several STEM images.

the particles. Therefore, the balls with less diameter compared to previous balls were introduced for the next grinding step. Then the particle size was further reduced and again reached equilibrium. Therefore, the sequential application of grinding with grinding balls of different size tends to form dispersion with narrow distribution of particle size.

\section{Conclusions}

Stable dispersion of oleic acid coated magnetite nanoparticles with uniform particle size distribution has been synthesized through top-down approach using a high purity ore available in Sri Lanka. The ore was wet-ground using 


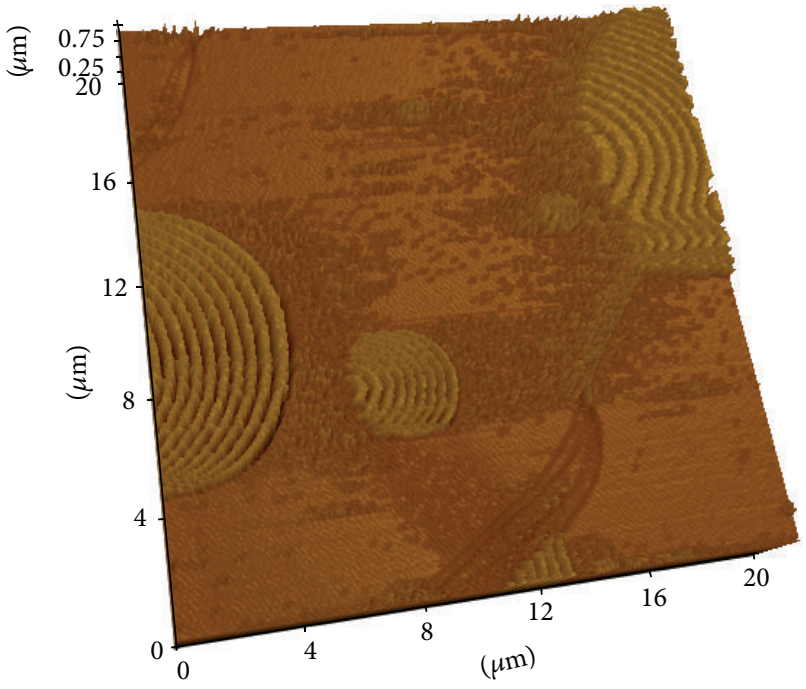

(a)

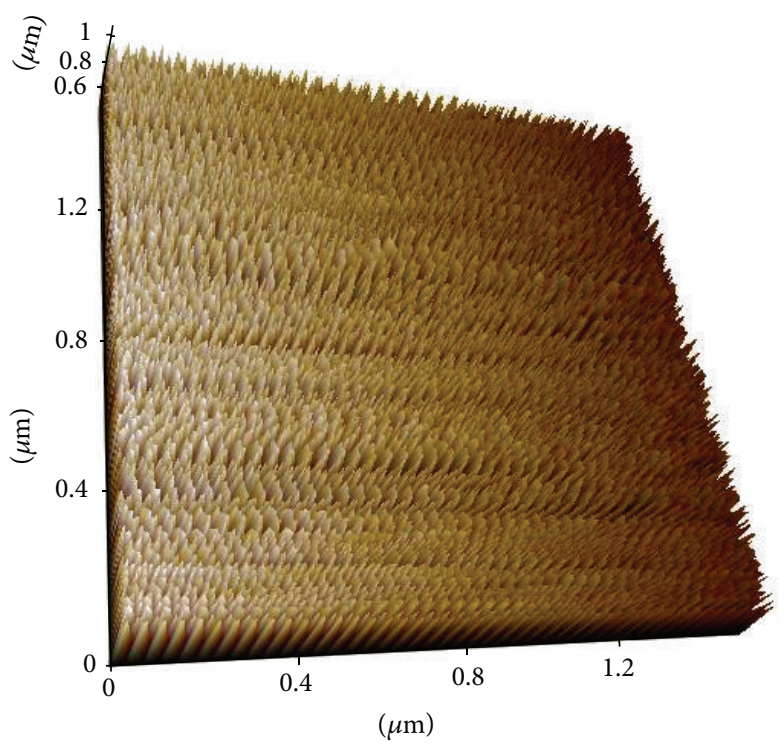

(b)

FIGURE 5: MFM images of (a) MAG-Ole-Grd-5 and (b) magnified image obtained using XE-100 atomic force microscope with a magnetic force microscopic attachment.

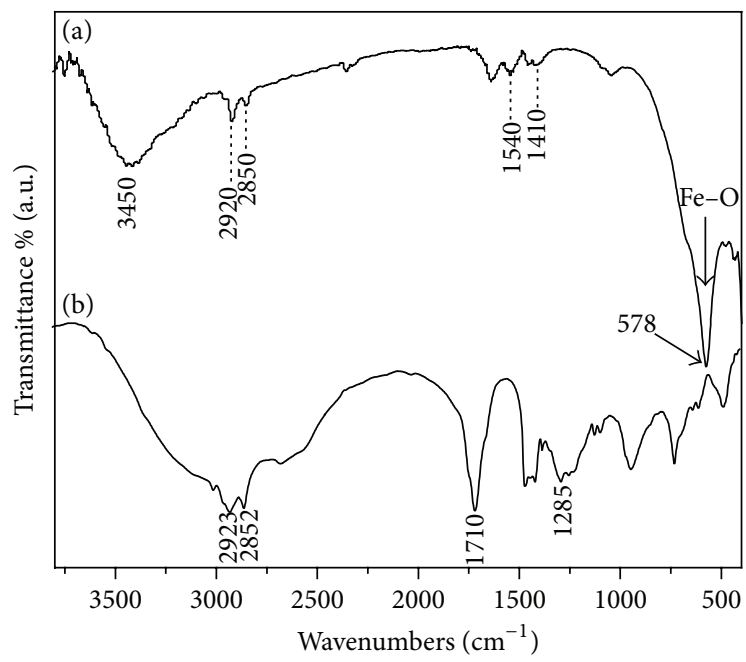

FIGURE 6: FTIR spectra of (a) MAG-Ole-Grd-5 and (b) pure oleic acid obtained using Bruker Vertex 80 coupled with Ram-FT module (RAM II) Fourier transform infrared spectrophotometer.

the FRITSCH Planeten-Mikromühle PULVERISETTE 7 premium line grinder in an inert atmosphere in the presence of oleic acid. By considering all the analytical methods such as particle size analysis, SEM imaging, STEM imaging, and AFM imaging, the particle size of the magnetite nanoparticles which resulted after final grinding step (MAG-Ole-Grd-5) is within the range of $20-50 \mathrm{~nm}$ and has a zeta potential value of $+42 \mathrm{mV}$. This experiment was repeated three times and the results were reproducible. Significantly, this synthesis of magnetite nanoparticles through top-down approach significantly reduced the grinding time to $5 \mathrm{hrs}$ compared to what was previously reported.

\section{Conflict of Interests}

The authors declare that there is no conflict of interests regarding the publication of this paper.

\section{Acknowledgments}

The authors acknowledge Geological Survey and Mines Bureau (GSMB), Sri Lanka, for providing magnetite ore samples and Mr. Sunanda Gunasekara for the technical support given throughout the experiment.

\section{References}

[1] A. A. Gungor, H. Nadaroglu, and N. Celebi, "Fenton process for basic red 9 degradation: immobilized apolaccase on a nanomagnetite system," Environmental and Experimental Biology, vol. 12, pp. 121-129, 2014.

[2] D. Mohan and C. U. Pittman Jr., "Arsenic removal from water/wastewater using adsorbents-a critical review," Journal of Hazardous Materials, vol. 142, no. 1-2, pp. 1-53, 2007.

[3] M. Hua, S. Zhang, B. Pan, W. Zhang, L. Lv, and Q. Zhang, "Heavy metal removal from water/wastewater by nanosized metal oxides: a review," Journal of Hazardous Materials, vol. 211212, pp. 317-331, 2012.

[4] J. W. Farrell, J. Fortner, S. Work et al., "Arsenic removal by nanoscale magnetite in Guanajuato, Mexico," Environmental Engineering Science, vol. 31, no. 7, pp. 393-402, 2014.

[5] C. T. Yavuz, J. T. Mayo, C. Suchecki et al., "Pollution magnet: nano-magnetite for arsenic removal from drinking water," Environmental Geochemistry and Health, vol. 32, no. 4, pp. 327334, 2010.

[6] J. Sun, S. Zhou, P. Hou et al., "Synthesis and characterization of biocompatible $\mathrm{Fe}_{3} \mathrm{O}_{4}$ nanoparticles," Journal of Biomedical Materials Research Part A, vol. 80, no. 2, pp. 333-341, 2007. 
[7] A. G. Roca, D. Niznansky, J. Poltierova-Vejpravova et al., "Magnetite nanoparticles with no surface spin canting," Journal of Applied Physics, vol. 105, no. 11, Article ID 114309, 2009.

[8] B. Duong, H. Khurshid, P. Gangopadhyay et al., "Enhanced magnetism in highly ordered magnetite nanoparticle-filled nanohole arrays," Small, vol. 10, no. 14, pp. 2840-2848, 2014.

[9] M. Ataeefard, E. Ghasemi, and M. Ebadi, "Effect of microand nanomagnetite on printing toner properties," The Scientific World Journal, vol. 2014, Article ID 706367, 7 pages, 2014.

[10] M. Shabanian, M. Khoobi, F. Hemati et al., "New PLA/PEIfunctionalized $\mathrm{Fe}_{3} \mathrm{O}_{4}$ nanocomposite: preparation and characterization," Journal of Industrial and Engineering Chemistry, vol. 24, pp. 211-218, 2015.

[11] H. B. Na, I. C. Song, and T. Hyeon, "Inorganic nanoparticles for MRI contrast agents," Advanced Materials, vol. 21, no. 21, pp. 2133-2148, 2009.

[12] J. Chomoucka, J. Drbohlavova, D. Huska, V. Adam, R. Kizek, and J. Hubalek, "Magnetic nanoparticles and targeted drug delivering," Pharmacological Research, vol. 62, no. 2, pp. 144$149,2010$.

[13] R. Y. Hong, T. T. Pan, and H. Z. Li, "Microwave synthesis of magnetic $\mathrm{Fe}_{3} \mathrm{O}_{4}$ nanoparticles used as a precursor of nanocomposites and ferrofluids," Journal of Magnetism and Magnetic Materials, vol. 303, no. 1, pp. 60-68, 2006.

[14] A. Z. Wilczewska, K. Niemirowicz, K. H. Markiewicz, and H. Car, "Nanoparticles as drug delivery systems," Pharmacological Reports, vol. 64, no. 5, pp. 1020-1037, 2012.

[15] F. Dong, W. Guo, J.-H. Bae, S.-H. Kim, and C.-S. Ha, "Highly porous, water-soluble, superparamagnetic, and biocompatible magnetite nanocrystal clusters for targeted drug delivery," Chemistry - A European Journal, vol. 17, no. 45, pp. 12802-12808, 2011.

[16] M. Mohapatra and S. Anand, "Synthesis and applications of nano-structured iron oxides/hydroxides-a review," International Journal of Engineering, Science and Technology, vol. 2, no. 8, pp. 127-146, 2011.

[17] M. B. Gawande, P. S. Branco, and R. S. Varma, "Nano-magnetite $\left(\mathrm{Fe}_{3} \mathrm{O}_{4}\right)$ as a support for recyclable catalysts in the development of sustainable methodologies," Chemical Society Reviews, vol. 42, no. 8, pp. 3371-3393, 2013.

[18] K. S. Wilson, L. A. Harris, J. D. Goff, J. S. Riffle, and J. P. Dailey, "A generalized method for magnetite nanoparticle steric stabilization utilizing block copolymers containing carboxylic acids," European Cells and Materials, vol. 3, no. 2, pp. 206-209, 2002.

[19] W. Wu, Q. He, and C. Jiang, "Magnetic iron oxide nanoparticles: synthesis and surface functionalization strategies," Nanoscale Research Letters, vol. 3, no. 11, pp. 397-415, 2008.

[20] Y.-K. Jeong, D.-K. Shin, H.-J. Lee, K.-S. Oh, J.-H. Lee, and D.-H. Riu, "Nano magnetite particles prepared under the combined addition of urea and ammonia," Key Engineering Materials, vol. 317-318, pp. 203-206, 2006.

[21] Z. Li, Q. Sun, and M. Gao, "Preparation of water-soluble magnetite nanocrystals from hydrated ferric salts in 2pyrrolidone: mechanism leading to $\mathrm{Fe}_{3} \mathrm{O}_{4}$," Angewandte Chemie-International Edition, vol. 44, no. 1, pp. 123-126, 2004.

[22] L. Zhang, R. He, and H.-C. Gu, "Oleic acid coating on the monodisperse magnetite nanoparticles," Applied Surface Science, vol. 253, no. 5, pp. 2611-2617, 2006.

[23] A. Khodabakhshi, M. M. Amin, and M. Mozaffari, "Synthesis of magnetite nanoparticles and evaluation of its efficiency for arsenic removal from simulated industrial wastewater," Iranian Journal of Environmental Health Science \& Engineering, vol. 8, no. 3, pp. 189-200, 2011.

[24] S. Xuan, L. Hao, W. Jiang, X. Gong, Y. Hu, and Z. Chen, "Preparation of water-soluble magnetite nanocrystals through hydrothermal approach," Journal of Magnetism and Magnetic Materials, vol. 308, no. 2, pp. 210-213, 2007.

[25] H. Karami and E. Chidar, "Pulsed-electrochemical synthesis and characterizations of magnetite nanorods," International Journal of Electrochemical Science, vol. 7, no. 3, pp. 2077-2090, 2012.

[26] F. Fajaroh, H. Setyawan, W. Widiyastuti, and S. Winardi, "Synthesis of magnetite nanoparticles by surfactant-free electrochemical method in an aqueous system," Advanced Powder Technology, vol. 23, no. 3, pp. 328-333, 2012.

[27] T. H. Nguyen, H. H. Nguyen, and H. L. Nguyen, "Effects of the conditions of the microemulsion preparation on the properties of Fe3O4 nanoparticles," Journal of Science VNU, Natural Sciences and Technology, vol. 24, pp. 9-15, 2008.

[28] Y. Mizukoshi, T. Shuto, N. Masahashi, and S. Tanabe, "Preparation of superparamagnetic magnetite nanoparticles by reverse precipitation method: contribution of sonochemically generated oxidants," Ultrasonics Sonochemistry, vol. 16, no. 4, pp. 525531,2009

[29] N. Chomchoey, D. Bhongsuwan, and T. Bhongsuwan, "Magnetic properties of magnetite nanoparticles synthesized by oxidative alkaline hydrolysis of iron powder," Kasetsart Journal: Natural Science, vol. 44, no. 5, pp. 963-971, 2010.

[30] W.-W. Wang, Y.-J. Zhu, and M.-L. Ruan, "Microwave-assisted synthesis and magnetic property of magnetite and hematite nanoparticles," Journal of Nanoparticle Research, vol. 9, no. 3, pp. 419-426, 2007.

[31] L. Almásy, D. Creanga, C. Nadejde, L. Rosta, E. Pomjakushina, and M. Ursache-Oprisan, "Wet milling versus co-precipitation in magnetite ferrofluid preparation," Journal of the Serbian Chemical Society, vol. 80, no. 3, pp. 367-376, 2015.

[32] S.-H. Back, G.-H. Lee, and S. Kang, "Effect of cryomilling on particle size and microstrain in a WC-Co alloy," Materials Transactions, vol. 46, no. 1, pp. 105-110, 2005.

[33] S. Vives, E. Gaffet, and C. Meunier, "X-ray diffraction line profile analysis of iron ball milled powders," Materials Science and Engineering A, vol. 366, no. 2, pp. 229-238, 2004.

[34] A. Mahmoud, H. Wasly, and M. Doheim, "Studies of crystallite size and lattice strain in $\mathrm{Al}-\mathrm{Al}_{2} \mathrm{O}_{3}$ powders produced by highenergy mechanical milling," Journal of Engineering Sciences, vol. 42, no. 6, pp. 1430-1439, 2014.

[35] A. N. Maratkanova, A. V. Syugaev, D. A. Petrov, and S. F. Lomayeva, "Structural characterization and microwave properties of chemically functionalized iron particles obtained by high-energy ball milling in paraffin-containing organic environment," Powder Technology, vol. 274, pp. 349-361, 2015.

[36] H. J. Jung, Y. Sohn, H. G. Sung, H. S. Hyun, and W. G. Shin, "Physicochemical properties of ball milled boron particles: dry vs. wet ball milling process," Powder Technology, vol. 269, pp. 548-553, 2015.

[37] S. Razavi-Tousi and J. Szpunar, "Effect of ball size on steady state of aluminum powder and efficiency of impacts during milling," Powder Technology, vol. 284, pp. 149-158, 2015.

[38] M. Muslimin and M. Yusoff, "The effect of high-energy milling on the crystallite size of alumina," Journal of Nuclear and Related Technologies, vol. 6, no. 1, pp. 95-102, 2009. 
[39] A. Senaratne, H. A. D. Dharmagunawardena, and G. W. A. R. Fernando, "Discovery of a new primary magnetite deposit in Wellawaya," in Proceedings of the Annual Research Sessions (PURSE '01), vol. 6, p. 133, Peradeniya, Sri Lanka, November 2001.

[40] H. Ahamed and V. S. Kumar, "A comparative study on the milling speed for the synthesis of nano-structured $\mathrm{Al} 6063$ alloy powder by mechanical alloying," Journal of Minerals and Materials Characterization \& Engineering, vol. 10, no. 06, pp. 507-515, 2011.

[41] T. J. Daou, S. Begin-Colin, J. M. Grenèche et al., "Phosphate adsorption properties of magnetite-based nanoparticles," Chemistry of Materials, vol. 19, no. 18, pp. 4494-4505, 2007.

[42] A. M. Atta, H. A. Al-Lohedan, and S. A. Al-Hussain, "Synthesis of stabilized myrrh-capped hydrocolloidal magnetite nanoparticles," Molecules, vol. 19, no. 8, pp. 11263-11278, 2014.

[43] N. Wu, L. Fu, M. Su, M. Aslam, K. C. Wong, and V. P. Dravid, "Interaction of fatty acid monolayers with cobalt nanoparticles," Nano Letters, vol. 4, no. 2, pp. 383-386, 2004. 

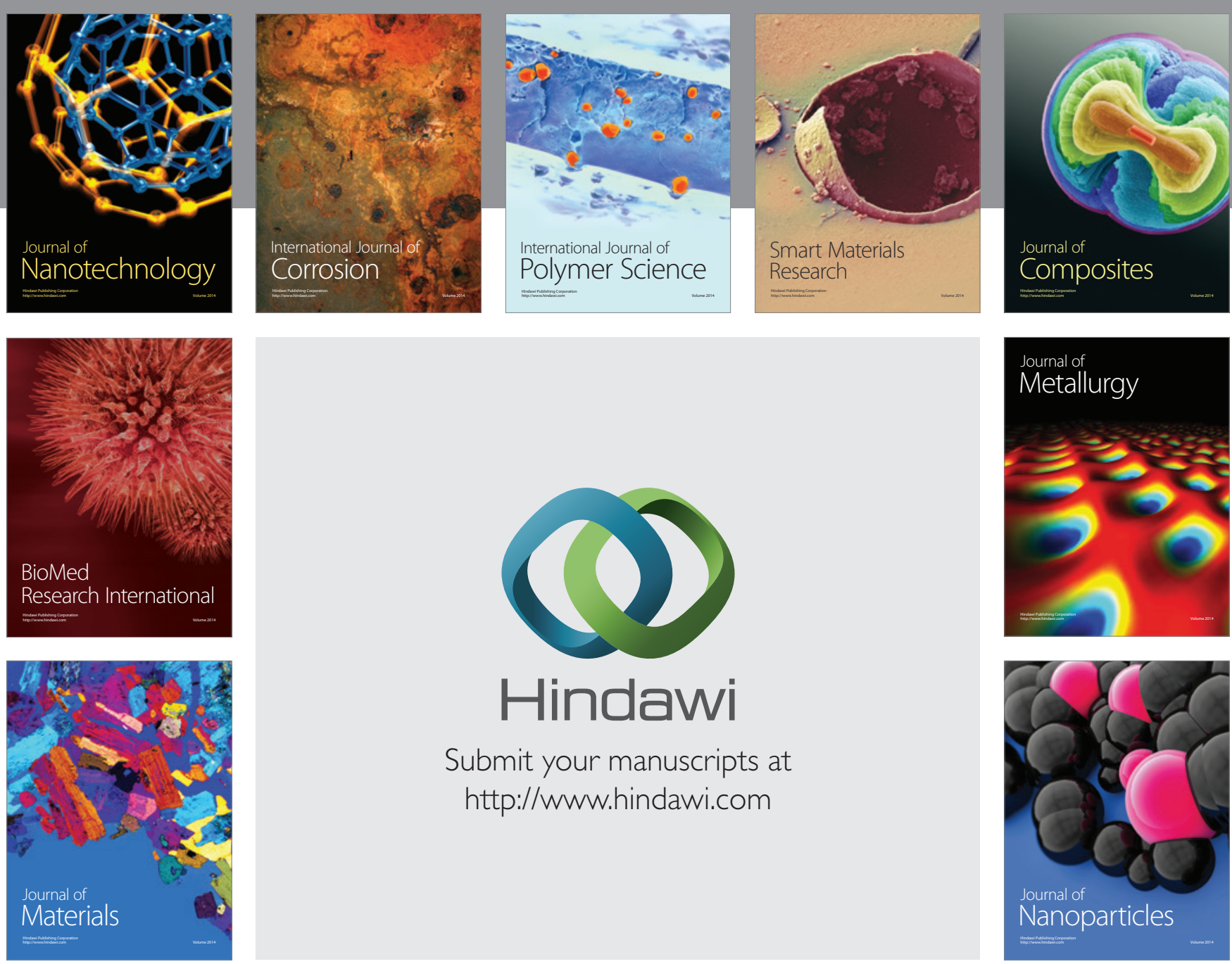

Submit your manuscripts at http://www.hindawi.com
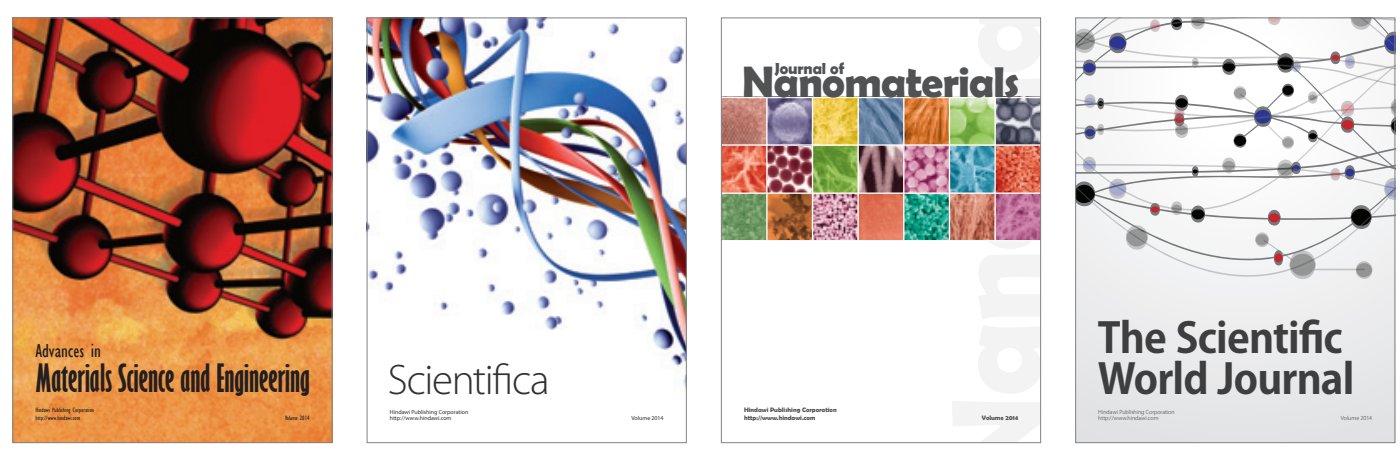

\section{The Scientific World Journal}
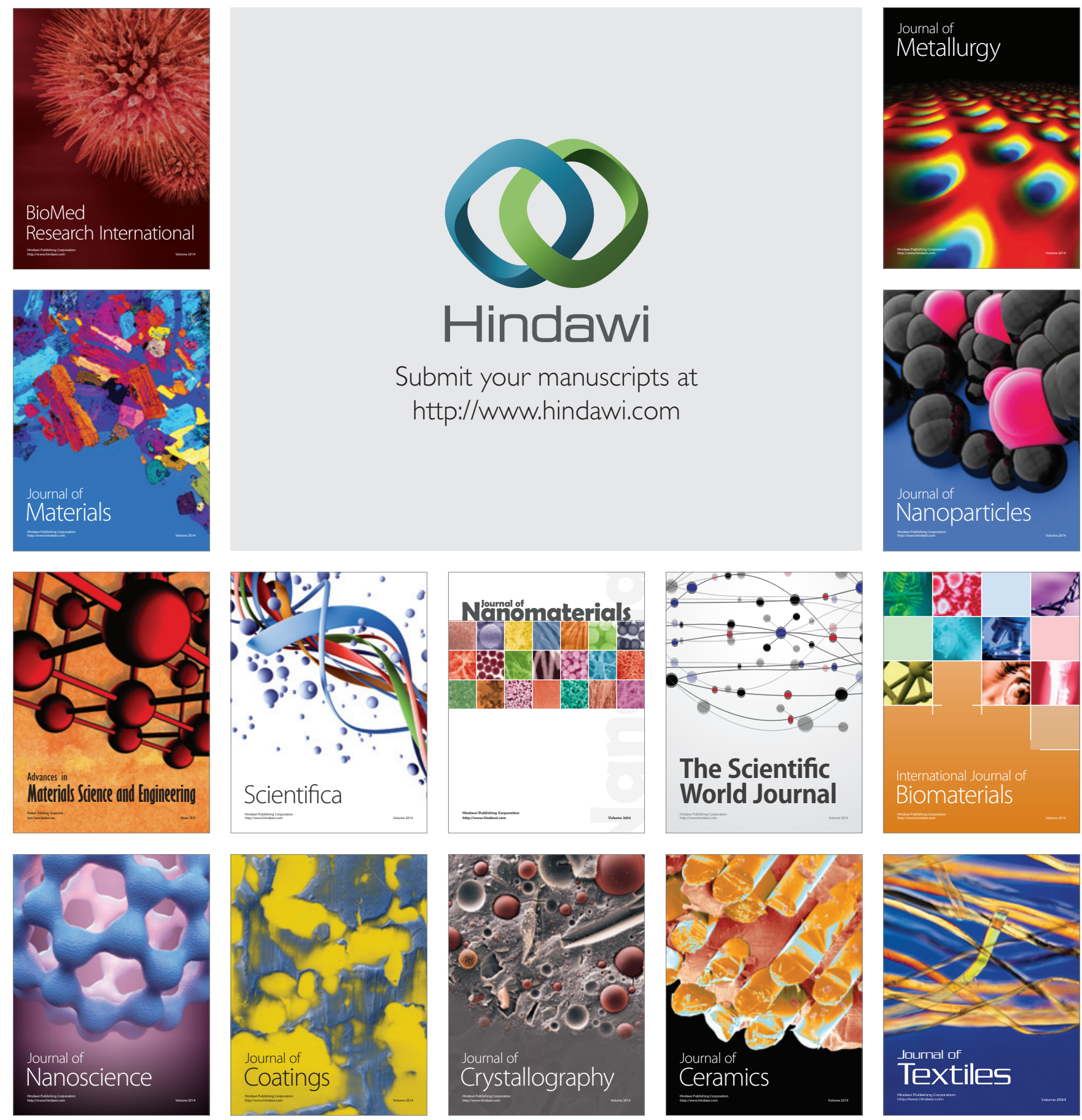